\title{
Nonstationarity of quasi-perpendicular shocks: a comparison of full particle simulations with different ion to electron mass ratio
}

\author{
M. Scholer and S. Matsukiyo \\ Max-Planck-Institut für Extraterrestrische Physik, 85741 Garching, Germany \\ Received: 21 November 2003 - Revised: 25 May 2004 - Accepted: 11 June 2004 - Published: 14 July 2004 \\ Part of Special Issue "Spatio-temporal analysis and multipoint measurements in space"
}

\begin{abstract}
We have performed 3 one-dimensional full particle electromagnetic simulations of a quasi-perpendicular shock with the same Alfvén Mach number $M_{A}=4.5$, shock normal - magnetic field angle $\Theta_{B n}=87^{\circ}$, and ion and electron beta (particle to magnetic field pressure) of 0.05 , but with different ion to electron mass ratios $\left(m_{i} / m_{e}=80,400\right.$, 1840). At high ion beta it has been shown previously that the shock is steady. At low ion beta, as in the present simulations, the shock periodically reforms itself. However, whereas at unrealistically low mass ratios the reformation is due to accumulation of specularly reflected particles at the upstream edge of the foot, at the realistic mass ratio the modified two-stream instability between the incoming solar wind ions and solar wind electrons leads to ion phase mixing and thermalization. The reformation process is thereby considerably modified. At the lowest mass ratio the Buneman instability between the solar wind electrons and the reflected ions is excited, which is stabilized at higher mass ratios.
\end{abstract}

Key words. Space plasma physics (kinetic and MHD theory; numerical simulation studies; shock waves)

\section{Introduction}

Beginning with Biskamp and Welter (1972) collisionless shocks have been investigated by full particle (Particle-InCell) simulations for more than three decades. It has been shown that perpendicular and quasi-perpendicular shocks are intrinsically instationary and considerable attention has been paid to the cyclic self-reformation (Biskamp and Welter, 1972; Lembège et al., 1987, 1992). This process is due to the fact that part of the upstream ions are reflected at the shock front and are responsible for the formation of the foot. The accumulation of these ions in time is responsible for the cyclic self-reformation with time scales of the order of the inverse ion gyrofrequency $\Omega_{c i}$. We will concentrate in this

Correspondence to: M. Scholer

(mbs@mpe.mpg.de) paper on quasi-perpendicular shocks with $\Theta_{B n}$ close to $90^{\circ}$. At shocks below a whistler critical Mach number given by

$M_{w}=\frac{1}{2}\left(\frac{m_{i}}{m_{e}}\right)^{1 / 2} \cos \Theta_{B n}$

a linear whistler train can phase-stand in the upstream flow in front of the shock (Kennel et al., 1985). Krasnoselskikh et al. (2002) have suggested that above a nonlinear whistler critical Mach number given by

$M_{n w}=\frac{1}{\sqrt{2}}\left(\frac{m_{i}}{m_{e}}\right)^{1 / 2} \cos \Theta_{B n}$

a nonlinear upstream whistler train becomes unstable to a gradient catastrophy, which is expected to lead to shock reformation. In the following we will concentrate on shocks well above the nonlinear whistler critical Mach number. For example, in the case of a shock with Alfvén Mach number $M_{A}=4$, one obtains $\Theta_{n w} \approx 82.5^{\circ}$ (assuming $m_{i} / m_{e}=1840$ ), above which a nonlinear wave train cannot exist anymore within the shock front.

Early full particle simulations, which revealed cyclic reformation of the shock front, were done for extremely small ion to electron mass ratios $(\leq 100)$ and for small ion and electron beta. Reformation in these simulations was due to accumulation of specularly reflected ions near the upstream edge of the foot once they become turned around in the course of their gyromotion. This accumulation leads to a density and magnetic field increase, and a feedback effect eventually leads to the emergence of a new shock front near the upstream edge of the foot. Schmitz et al. (2002) first noted that the reformation process disappears in PIC simulations of higher beta shocks ( $\beta_{i}$ of the order of 1), a result which was later confirmed by Scholer et al. (2003) and by Hada et al. (2003). Scholer et al. (2003) used the realistic ion to electron mass ratio and found that indeed in the regime $\beta_{i} \geq 0.4$ the reformation process disappears. At low ion beta $\left(\beta_{i}=0.2\right)$ a reformation occurred as described earlier in the low mass ratio simulations by, for example Biskamp and Welter (1972) 
and Lembège et al. $(1987,1992)$. However, in between reformation cycles a large part of the incident ions is specularly reflected at the shock potential. As these ions propagate upstream away from the shock and constitute a foot the incoming electrons are decelerated in order to achieve zero electrical current in the shock normal direction. In a configuration which is not exactly perpendicular, i.e. when a component of wave vectors parallel to the magnetic field is allowed, the resulting difference between incoming ions and incoming electrons leads to the excitation of the electromagnetic modified two-stream instability. The waves generated by this instability are on the whistler mode branch and slightly modify the shock profile. Since the shock is slightly oblique the normal wave electric field has a component parallel to the magnetic field which leads to acceleration and trapping, and eventually to a pre-heating of the electrons in the foot of the shock parallel to the magnetic field. This is quite different from the preheating of electrons perpendicular to the magnetic field in the foot of perpendicular shock in the waves excited by the Buneman instability (Buneman, 1958), as obtained by Shimada and Hoshino (2000) and Schmitz et al. (2002) in PIC simulations of exactly perpendicular shocks.

Hada et al. (2003) have recently proposed a semianalytical model for the shock reformation process, which is based on the coupling between incoming and reflected ions. They determine a critical fraction of reflected to incoming ions beyond which no stationary solution for the coupling process exists. In other words, for a high reflection rate the coupling becomes so strong that the shock is nonstationary and reforms. As pointed out by Hada et al. (2003) the proposed model applies for a large Mach number range, but is restricted to the case where the reflected ions can be described by a mono-ion population, which occurs for low ion beta. Scholer et al. (2003) suggested that the important quantity determining whether reformation occurs or not is actually not the ion beta, but the difference between the upstream plasma velocity and the ion thermal velocity: when this difference is large the incoming and reflected ion beam interact upstream at the beginning of the foot, and this interaction starts a reformation process. When this difference is small, as in $\beta_{i} \approx 0.4$ simulations of medium Mach number shocks, the incoming and reflected ion beams are overlapping in velocity space in the region close to the shock ramp. The interaction then occurs smoothly over the whole foot and a stationary profile results.

The Hada et al. (2003) model thus seems only to work (in the case of medium Mach number shocks) in the low ion beta regime for exactly $\Theta_{B n}=90^{\circ}$ shocks or else when the modified two-stream instability is not excited. However, as already seen in the Scholer et al. (2003) simulations and from an evaluation of the linear theory for parameters appropriate to the foot of quasi-perpendicular shocks by Matsukiyo and Scholer (2003) the modified two-stream instability can modify the reformation and heating process for shocks with a small, but finite deviation of $\Theta_{B n}=90^{\circ}$, since the instability produces waves with a wave vector component parallel to the magnetic field. We note that a direct comparison with the
Hada et al. (2003) analysis is not possible at this point, since the latter is done for $\Theta_{B n}=90^{\circ}$. Investigation of this process requires simulations with realistic ion to electron mass ratio, since the growth rate of the modified two-stream instability in units of the inverse ion gyrofrequency depends strongly on $m_{i} / m_{e}$ (Matsukiyo and Scholer, 2003). In this paper we will investigate the reformation process for a low ion beta shock by a one-dimensional PIC simulation. Using the same values of Mach number and $\Theta_{B n}$ we will vary the ion to electron mass ratio. The sobering fact is that not only the physical mechanism leading to nonstationarity depends critically on the mass ratio, but also the mechanism leading to electron heating in the shock is strongly mass ratio dependent. We conclude that while unrealistic mass ratio simulations will, under certain conditions, give insight into shock related processes, taking the realistic mass ratio into account may modify the detailed physical processes.

\section{Simulation}

The shock is produced by the so-called injection method: a high-speed plasma consisting of electrons and ions is injected from the left-hand boundary of a one-dimensional simulation system and travels toward positive $x$. The plasma carries a uniform magnetic field which has a $B_{z}$ and a $B_{x}$ component. At the right-hand boundary the particles are specularly reflected. A shock then propagates in the $-x$ direction, i.e. the simulation system is the downstream rest frame, and the shock normal is the $\mathrm{x}$-axis. Furthermore, the simulations are done in the so-called normal incidence frame where the upstream bulk velocity is parallel to the shock normal. Initially there are 80 particles for each species in a computational cell; the simulation box consists of 9000 grid cells, each of the size of one Debye length $\lambda_{D}$. In the following, time will be given in units of the inverse of the ion cyclotron frequency $\Omega_{c i}$, distances in units of the electron inertial length $\lambda_{e}$, the velocity in units of the upstream Alfvén speed $v_{A}$, magnetic field and the density in units of their upstream values $B_{0}$ and $n_{0}$, respectively. The potential $e \Phi$ is given in units of $c B_{0} / \lambda_{e}$. We use $\Theta_{B n}=87^{\circ}$ and inject the plasma with Alfvén Mach number $M_{A 0}=3.0$ which leads to a $M_{A} \sim 4.5$ shock. As outlined in the Introduction, we will investigate a low beta case and assume $\beta_{i}=\beta_{e}=0.05$. Because of computational reasons the parameter $\left(\omega_{p e} / \Omega_{c e}\right)$ is set to 2 . Note that in the solar wind at the Earth's orbit this quantity is 100-200. When we use an ion to electron mass ratio of $m_{i} / m_{e}=1840$ a 1D shock run up to time $t \Omega_{c i} \sim 3$ takes on a 4 processor machine about $400 \mathrm{~h}$. The computational effort increases with the fourth power of $\omega_{p e} / \Omega_{c e}$, from which it can be seen that increasing this parameter in shock simulations much further and keeping at the same time the realistic mass ratio are not yet feasible. For the present investigation we adopt the philosophy that the mass ratio is a physical given quantity, whereas the ratio of the two electron frequencies can vary according to the astrophysical setting. Furthermore, as shown by Matsukiyo and Scholer (2003), the growth rate of 


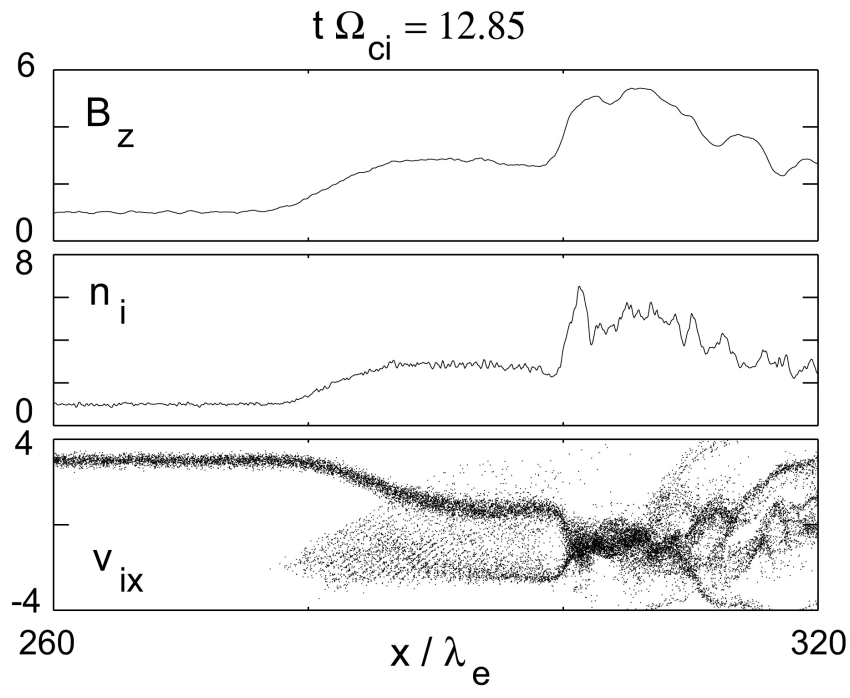

Fig. 1. From top to bottom: magnetic field $B_{z}$ component, ion density $n_{i}$, and ion $v_{i x}$ phase space versus shock normal direction $x$ for the ion to electron mass ratio 80 run.

the modified two-stream instability depends only weakly on $\omega_{p e} / \Omega_{c e}$. Thus, we rather use a realistic mass ratio and assume the value of $\tau=\left(\omega_{p e} / \Omega_{c e}\right)^{2}=4$, which means that we are in the strongly magnetized limit. We will return to the problem of small $\tau$ in the Discussion section. In the following we first present a run with low mass ratio $m_{i} / m_{e}=80$ followed by a run with the higher mass ratio $m_{i} / m_{e}=400$ while keeping all other parameters the same. Finally, we present a run with realistic mass ratio $m_{i} / m_{e}=1840$. Note that in the past most 1-D shock simulations, with the exception of Liewer et al. (1991) and Scholer et al. (2003), have been done for mass ratios of 200 or less. Two-dimensional shock simulations have up to now, because of computer resources, only been performed for mass ratios up to 42 (Savoini and Lembège, 2001).

\subsection{Low mass ratio shock simulation $\left(m_{i} / m_{e}=80\right)$}

Ions specularly reflected at the quasi-perpendicular shock and returning to it create the foot of the shock. The interaction between the reflected ions and the solar wind electrons can lead to the Bunemann instability which results in Langmuir turbulence in the foot. The reflected ion beam is Buneman unstable if the relative velocity between the ion beam and the solar wind electrons is larger than the thermal velocity $v_{\mathrm{e}, \text { th }}$ of the electrons. Requiring zero electrical current in the shock normal direction in the foot results in a deceleration of the incoming solar wind electrons. The deceleration depends on the ratio of reflected to incoming ions: in case all ions are reflected the incoming electrons have zero velocity. As will be shown later, reflection rates in the simulations are at the beginning of a reformation cycle $\sim 50 \%$, so that the electron bulk velocity in the foot is $\sim 1 / 3$ of the far upstream electron bulk velocity when requiring zero current in

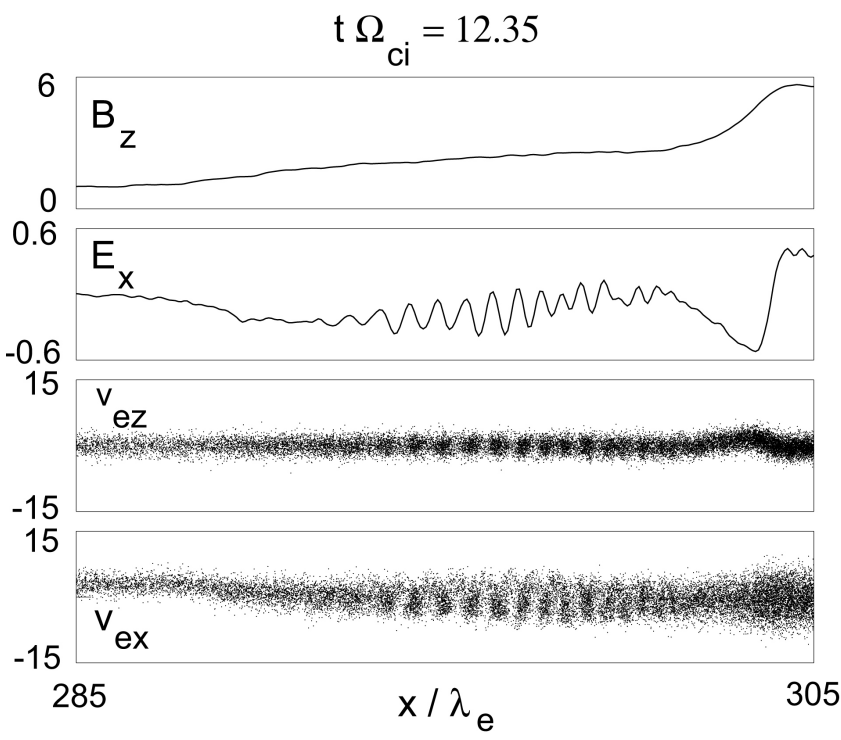

Fig. 2. From top to bottom: magnetic field $B_{z}$ component, electric field component $E_{x}$, and electron $v_{e z}$ and $v_{e x}$ phase space versus shock normal direction $x$.

the foot. The relative velocity $u_{\text {rel }}$ between specularly reflected ions and the incoming solar wind electrons in the foot is then $(4 / 3) U_{1}$, where $U_{1}$ is the upstream, or equivalently, the shock velocity. With the Alfvén Mach number $M_{A}$ and the electron beta $\beta_{e}$ the condition for the Buneman instability to occur can then be written

$\frac{\left(4 M_{A} / 3\right)^{2}}{\beta_{e}} \frac{m_{e}}{m_{i}}>1$.

Note that for the Buneman instability the wave frequency is larger than the gyrofrequency of the electrons and the wavelength is larger than the electron gyroradius, i.e. the electrons are unmagnetized. With the present shock parameters we obtain from Eq. (3) that for a mass ratio sufficiently below $\sim 720$ the Buneman instability should occur. At a higher mass ratio, or even at a mass ratio of the same order as this critical value, this instability is stabilized by Landau damping, since then the electron thermal velocity is larger than or comparable to the velocity difference between electrons and reflected ions. Vice versa, for the realistic mass ratio the Buneman instability does not occur unless the electron beta is less than $\sim 0.02$. Low beta, low mass ratio shock simulations are thus expected to result in Langmuir turbulence and rapid electron heating, which would be purely artificial.

Figure 1 shows, from top to bottom, the magnetic field $B_{z}$ component, the ion density $n_{i}$ and the ion $v_{i x}-x$ phase space plot at one particular time of the simulation $\left(t \Omega_{c i}=12.85\right)$. The unit scale in the shock normal direction $(x)$ is the electron inertial length, $\lambda_{e}$. The shock ramp is at $\sim 300 \lambda_{e}$; there is an extended foot region with specularly reflected ions in which the incoming solar wind ions are decelerated and the magnetic field is increased. This foot eventually develops into a new shock ramp, as will be shown for the case of 


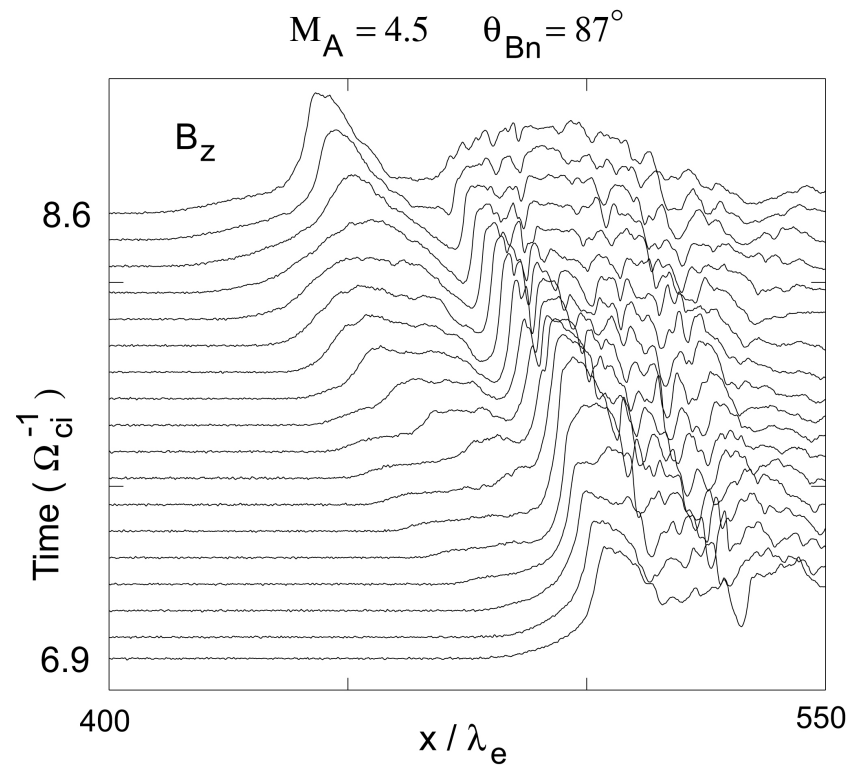

Fig. 3. Magnetic field $B_{z}$ component stacked in time for the ion to electron mass ratio 400 run. Time runs from bottom to top.

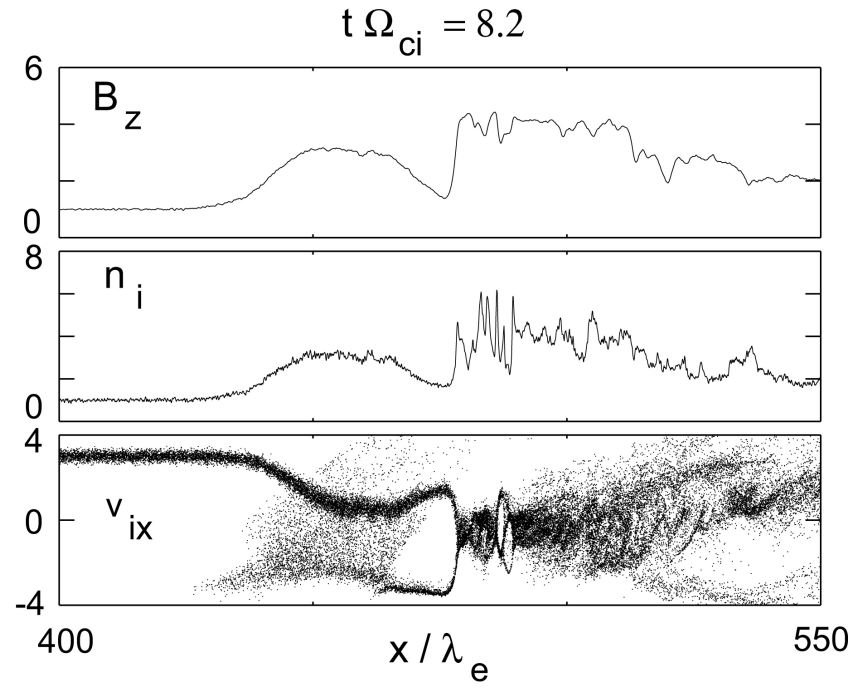

Fig. 4. From top to bottom: magnetic field $B_{z}$ component, ion density $n_{i}$, and ion $v_{i x}$ phase space versus shock normal direction $x$ for the ion to electron mass ratio 400 run.

the somewhat higher mass ratio $m_{i} / m_{e}=400$. Here, we are mainly interested in the electrons in the foot region. Figure 2 shows, on an expanded scale, the magnetic field component $B_{z}$, the electric field $E_{x}$ in the shock normal direction, and two components of the electron phase space, $v_{e z}-x$ and $v_{e x}-x$. Note that $v_{e x}$ is close to the velocity perpendicular to the magnetic field, whereas $v_{e z}$ is very close to the component parallel to the magnetic field. The $E_{x}$ component exhibits electrostatic waves in the foot region which lead to electron trapping and subsequent heating perpendicular to the magnetic field (bottom panel of Fig. 2). This is similar to what has been described at higher Mach number $\left(M_{A}=10\right)$ by Schmitz et al. (2002).

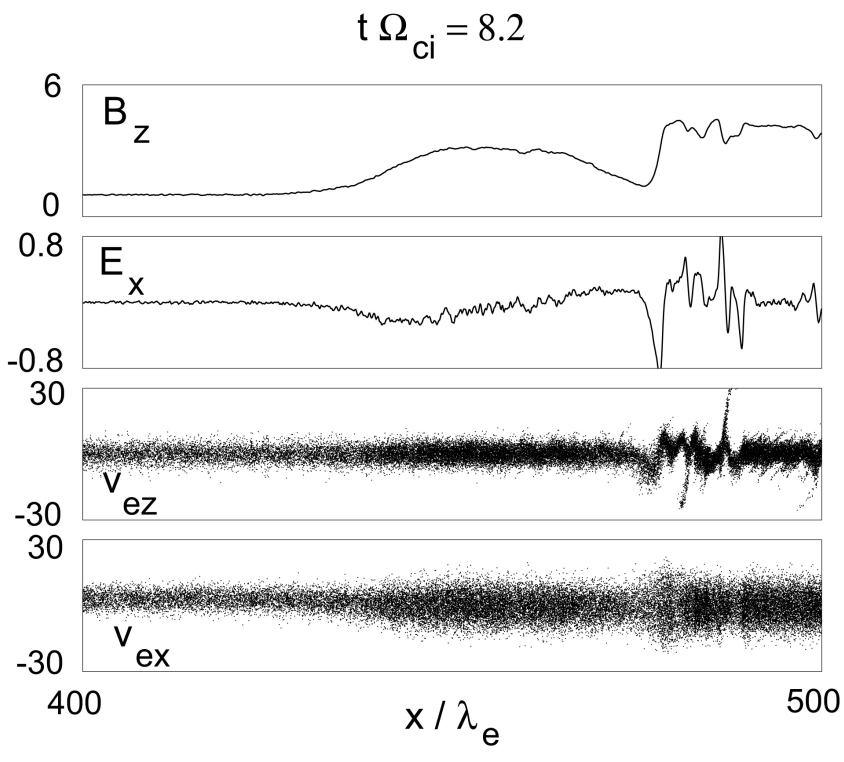

Fig. 5. From top to bottom: magnetic field $B_{z}$ component, electric field component $E_{x}$, and electron $v_{e z}$ and $v_{e x}$ phase space versus shock normal direction $x$.

\subsection{Medium mass ratio shock simulation $\left(m_{i} / m_{e}=400\right)$}

We now increase the ion to electron mass ratio to 400 . Figure 3 shows the magnetic field component $B_{z}$ stacked in time; time runs from bottom to top, and beginning and end is indicated at the y-axis. At $t \Omega_{c i}=6.9$ a sharp ramp can be seen. As time proceeds a foot builds up in front of the ramp and the foot magnetic field increases in magnitude. By $t \Omega_{c i}=8.6$ a new shock ramp has emerged at the upstream edge of the foot. This process, called self-reformation, repeats itself every $1-2 \Omega_{c i}^{-1}$ and has been described earlier (Biskamp and Welter, 1972; Lembège et al., 1987, 1992).

Figure 4 shows the magnetic field, the ion density, and ion phase space at one particular time during the reformation cycle in the same format as Fig. 1. The incoming solar wind ions are strongly decelerated in the foot. The accumulation of specularly reflected ions near the upstream edge of the foot where they are turned around in the magnetic field leads to a density increase, accompanied by a magnetic field increase. This results in further deceleration of the incoming solar wind ions, and the feedback effect eventually leads to a newly emerging shock front. This is similar as described in the reformation model for perpendicular shocks proposed by Hada et al. (2003) .

As can be seen from the electric field shown in Fig. 5 no electrostatic waves are generated in the foot region and the electron phase space plots show adiabatic electron heating and acceleration in the cross shock potential in the ramp. From Eq. (3) one would still expect the Buneman instability to occur in the foot. The absence of the instability at this medium mass ratio may be explained by Landau damping: the mass ratio 400 is of the same order as the critical value 720 . In addition, the actual velocity difference between 


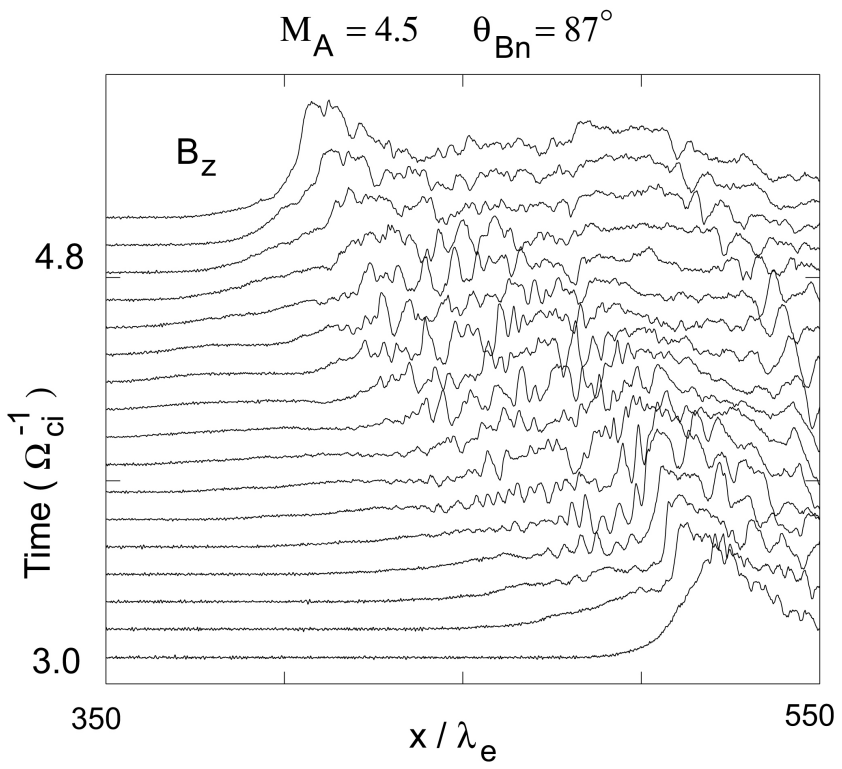

Fig. 6. Magnetic field $B_{z}$ component stacked in time for the ion to electron mass ratio 1840 run. Time runs from bottom to top.

electrons and reflected ions in the simulation is smaller than $(4 / 3) U_{1}$, because the incoming ions are already decelerated in the foot region. This further decreases the critical mass ratio.

\subsection{Realistic mass ratio shock simulation}

Scholer et al. (2003) have already shown that realistic mass ratio simulations of highly oblique quasi-perpendicular shocks at low ion beta also result in shock reformation. The purpose here is to demonstrate that the reformation mechanism is different from the mechanism leading to reformation in the low mass ratio runs and also different from the mechanism proposed by Hada et al. (2003). As we will demonstrate in the following, reformation is due to the excitation of the modified two-steam instability (MTSI) in the foot and subsequent phase-mixing and thermalization between incoming solar wind ions and specularly reflected ions. Figure 6 shows the magnetic profiles during one reformation cycle in the same format as Fig. 3. At the beginning of the cycle $\left(t \Omega_{c i} \sim 3.1\right)$ and at the end $\left(t \Omega_{c i}=4.7\right)$ the magnetic field exhibits a sharp ramp, while in between the field in the foot strongly fluctuates. The ion phase space plot in the bottom panel of Fig. 7 shows structure in the phase space distribution of the incoming ions. These ions interact with the incoming electrons: due to the high density of reflected ions the incoming electrons are decelerated relative to the incoming ions in order to achieve zero current in the shock normal direction. Due to the relative velocity between solar wind ions and solar wind electrons, the solar wind ion beam mode can interact with the whistler mode, as shown in more detail by Matsukiyo and Scholer (2003), which results in the MTSI. Figure 8 is a close-up of the foot region. Note that the scale corresponds to not much more than one ion inertia length.

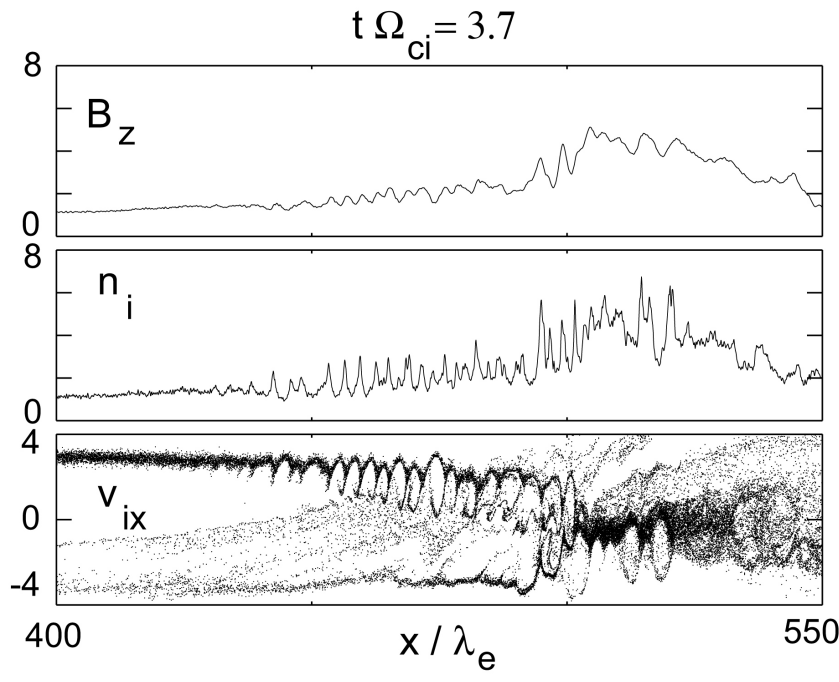

Fig. 7. From top to bottom: magnetic field $B_{z}$ component, ion density $n_{i}$, and ion $v_{i x}$ phase space versus shock normal direction $x$ for the ion to electron mass ratio 1840 run at $t \Omega_{c i}=3.7$.

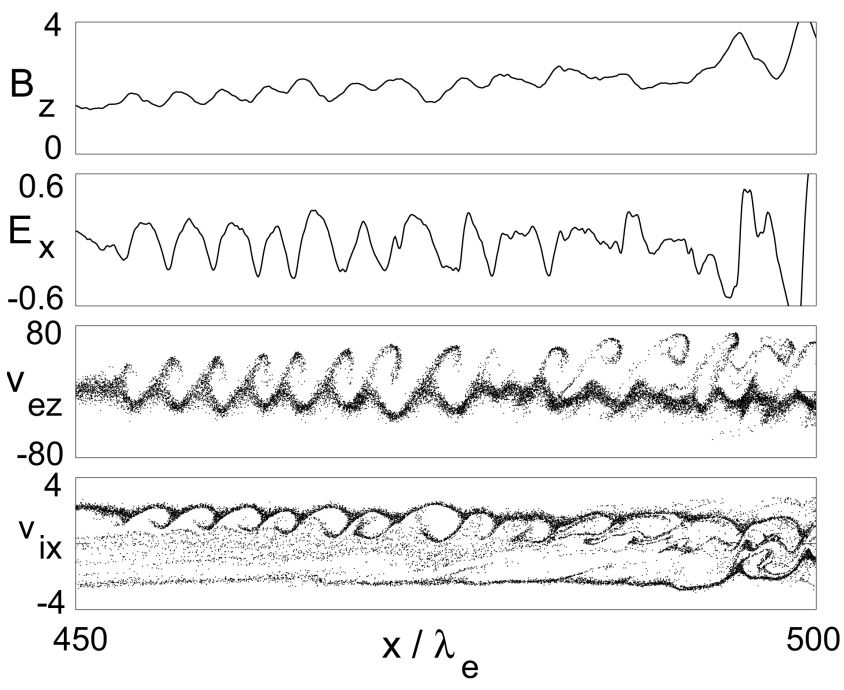

Fig. 8. From top to bottom: magnetic field $B_{z}$ component, electric field $E_{x}$ component, electron $v_{e z}$ phase space distribution and ion $v_{i x}$ phase space distribution in the foot of the shock $\left(m_{i} / m_{e}=1840\right.$ run) at $t \Omega_{c i}=3.7$.

It shows, in addition to the magnetic field and the ion phase space distribution in the top and bottom panel, respectively, the electric field component $E_{x}$ and the electron phase space distribution in the $z$ direction, i.e. almost parallel to the magnetic field. The electric field has a component parallel to the magnetic fields and results in electron trapping and acceleration.

If the electromagnetic waves in the foot are due to the MTSI based on the electron-incoming ion interaction the wave vectors have to be directed downstream. Figure 9 is 

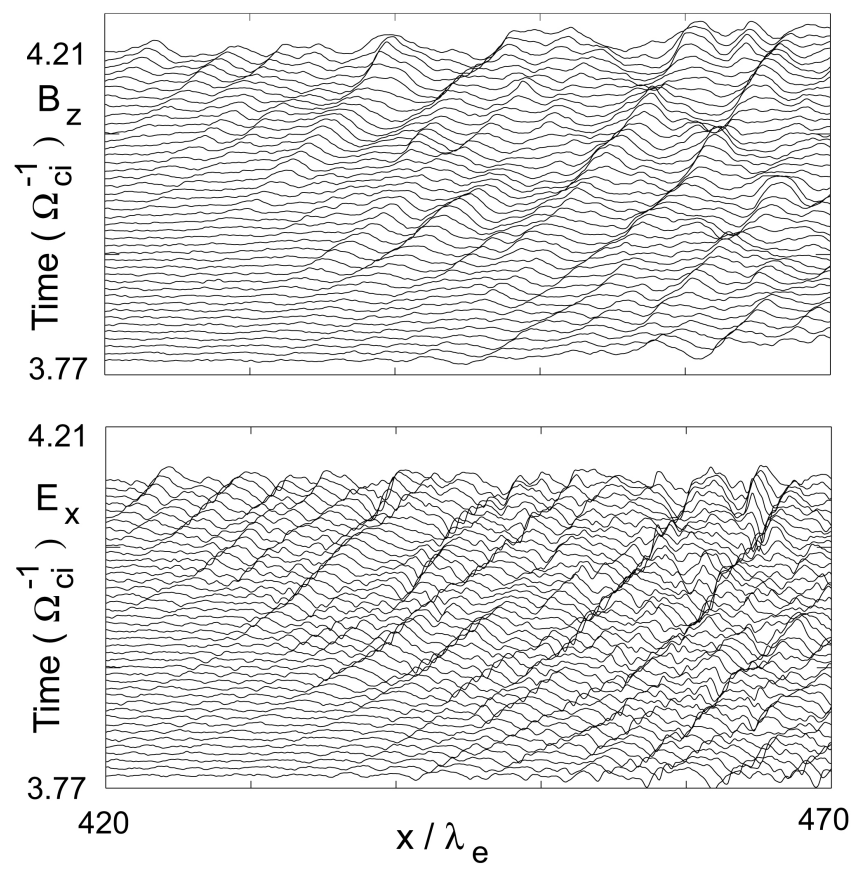

Fig. 9. Electric field $E_{x}$ component stacked in time. Time runs from bottom to top.

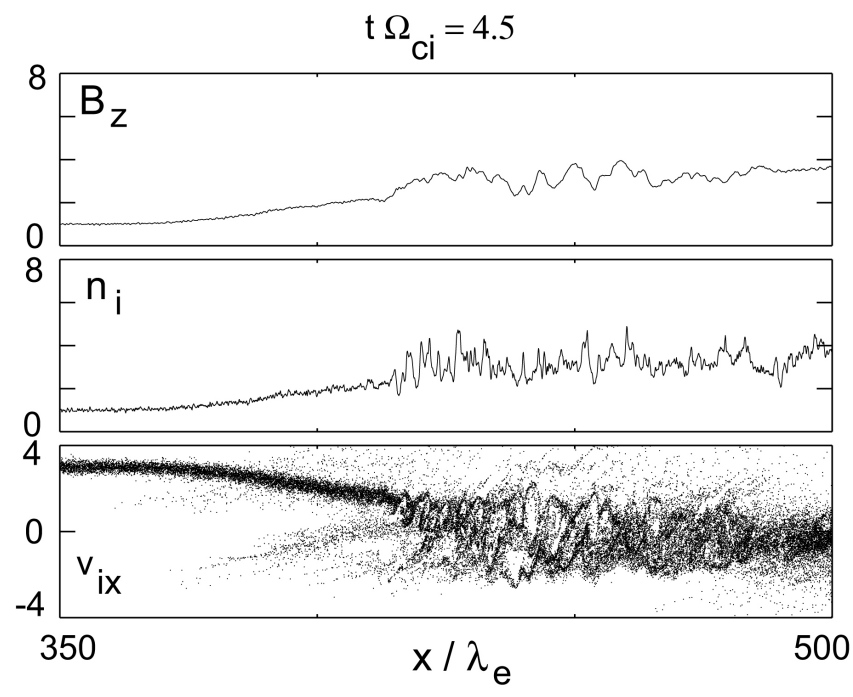

Fig. 10. Same as Fig. 10 at at $t \Omega_{c i}=4.5$.

a stack plot of the magnetic field $B_{z}$ component (top) and the electric field $E_{x}$ component over a short time interval $\left(0.44 \Omega_{c i}^{-1}\right)$ in the foot region. Beyond $x \sim 470 \lambda_{e}$ the ion and electron bulk velocity is almost zero in the downstream rest frame (simulation frame); between $x=420 \lambda_{e}$ and $x \sim 470 \lambda_{e}$ the velocity is less than $1 M_{A}$. From the slopes of the wave crests it can be seen that the waves originate at the upstream edge of the foot. The wave amplitude increases as they propagate in the bulk frame in the shock direction. This excludes dispersive effects propagating from the ramp as the cause for the waves in the foot region, which have been discussed

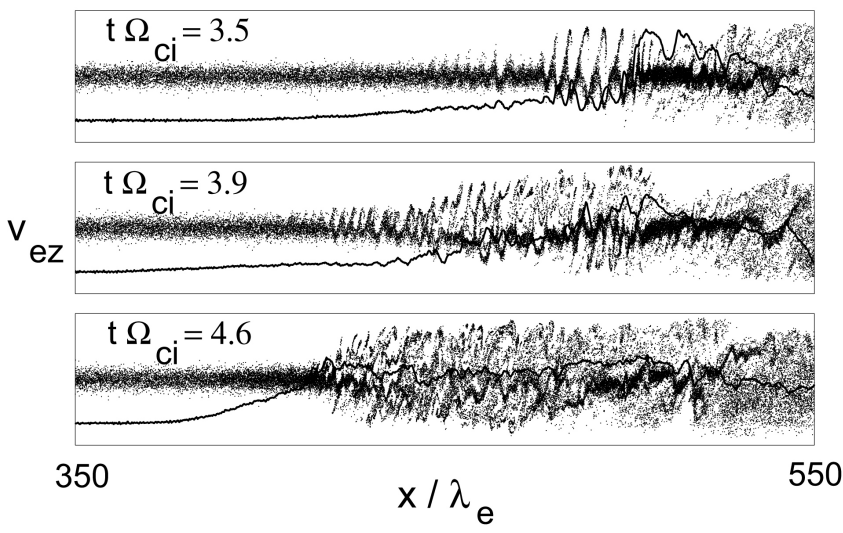

Fig. 11. Electron $v_{e z}-x$ phase space at three different times during a reformation cycle. Superposed are the $B_{z}$ magnetic field trace.

by Krasnoselskikh et al. (2002). Initially, the reflected ions do not participate directly in the development of the MTSI. However, the phase mixing of the reflected ions and the incoming ions due to the MTSI turbulence leads to solar wind ions thermalization in the foot region. Eventually, a new shock ramp emerges at the upstream edge of the foot, as can be seen from Fig. 10, which is the equivalent to Fig. 7 about $0.8 \Omega_{c i}^{-1}$ later. Note that there is no feedback effect due to specularly reflected ion accumulation at the upstream edge of the foot. We have determined the ratio of reflected ions to incoming solar wind ions in a $\left(20 \lambda_{e}\right.$ wide) region in the foot close to the ramp. Early in the reformation process (at $t=3.4 \Omega_{c i}^{-1}$ ) this ratio is $\sim 0.53$, i.e. about half of the incoming ions are reflected. The major part of these ions contributes, after reformation, to the hot core, whereas only a small part survives as downstream gyrating ions. The time $T_{c}$ for the reformation cycle is $T_{c} \sim 1.6 \Omega_{c i}^{-1}$. We have repeated the physical mass ratio run for $\Theta_{B n}=90^{\circ}$, where the MTSI is excluded by design. In this case the time for one reformation cycle was $T_{c} \sim 2.1 \Omega_{c i}^{-1}$, which also demonstrates the importance of the MTSI for the reformation process.

Electron trapping and heating during reformation can be seen from Fig. 11. Here we show electron $v_{e z}$ versus $x$ phase space at three different times during the reformation cycle. Superposed in each panel is a $B_{z}$ trace of the magnetic field. Since the magnetic field is almost perpendicular to the shock normal ( $x$ direction) $v_{e z}$ is to a good approximation the velocity of the electrons parallel to the magnetic field. As can be seen from the top panel, early in the reformation cycle, the incoming electrons are accelerated close to the ramp parallel to the magnetic field and are trapped. The wave activity progressively extends in the foot further upstream, with the electron acceleration region moving upstream (middle panel) as well. At the end of the reformation cycle phase mixing results in parallel heating in the whole foot region. It is important to note that parallel electron acceleration begins initially near the ramp and then proceeds more and more to the upstream edge of the foot. 


\section{Conclusions}

We have presented in this paper 3 full particle simulations of a quasi-perpendicular shock with the same Alfvén Mach number $M_{A}$ and shock normal - magnetic field angle $\Theta_{B n}$ but different ion to electron mass ratios. $M_{A}$ and $\Theta_{B n}$ are such that a nonlinear whistler wave train cannot phase-stand in front of the shock. In Scholer et al. (2003) we have already shown that higher ion beta $\left(\beta_{i}>0.4\right)$, almost perpendicular shocks at medium Mach numbers $\left(M_{A} \sim 5\right)$, do not exhibit reformation. In the present paper we have investigated a lower ion beta shock. We show that the mass ratio has a drastic effect on the physics which comes out of the simulation: using different ion to electron mass ratios, while keeping all other parameters the same, leads to completely different processes concerning ion and electron heating, and considerably modifies the process of shock reformation. The results of the present study can be summarized as follows.

1. At all three mass ratios the shock is nonstationary and reforms with a time scale of 1-2 inverse ion gyrofrequencies.

2. Due to the small electron thermal velocity at the small mass ratio of 80 the Buneman instability is not stabilized. The Langmuir turbulence leads to electron heating perpendicular to the magnetic field.

3. At a mass ratio of 400 the Buneman instability is stabilized; electrons are adiabatically heated and accelerated perpendicular to the magnetic field by the cross shock potential.

4. At both unrealistically low mass ratios reformation occurs due to accumulation of specularly reflected ions at the upstream edge of the foot. A feedback effect leads to a magnetic field increase, further deceleration of incoming ions and density increase, and eventually to a new shock ramp. This mechanism has been described by a semi-analytical model for exactly perpendicular shocks by Hada et al. (2003).

5. At the realistic mass ratio the modified two-stream instability is excited in the foot due to the interaction between the slowed down solar wind electrons and the solar wind ions. The phase mixing of solar wind ions and specularly reflected ions leads to a heating of the ions in the foot and a new ramp occurs upstream of the region of thermalized solar wind and reflected ions.

6. At the realistic mass ratio the electrons are accelerated and trapped parallel the magnetic field. This results in parallel electron heating.

While at any mass ratio the low ion beta, almost perpendicular shock is nonstationary where the details are different, depending on the mass ratio. At low mass ratio the kinetic instability discussed here is absent because of the small growth rate. Reformation is induced by the accumulation
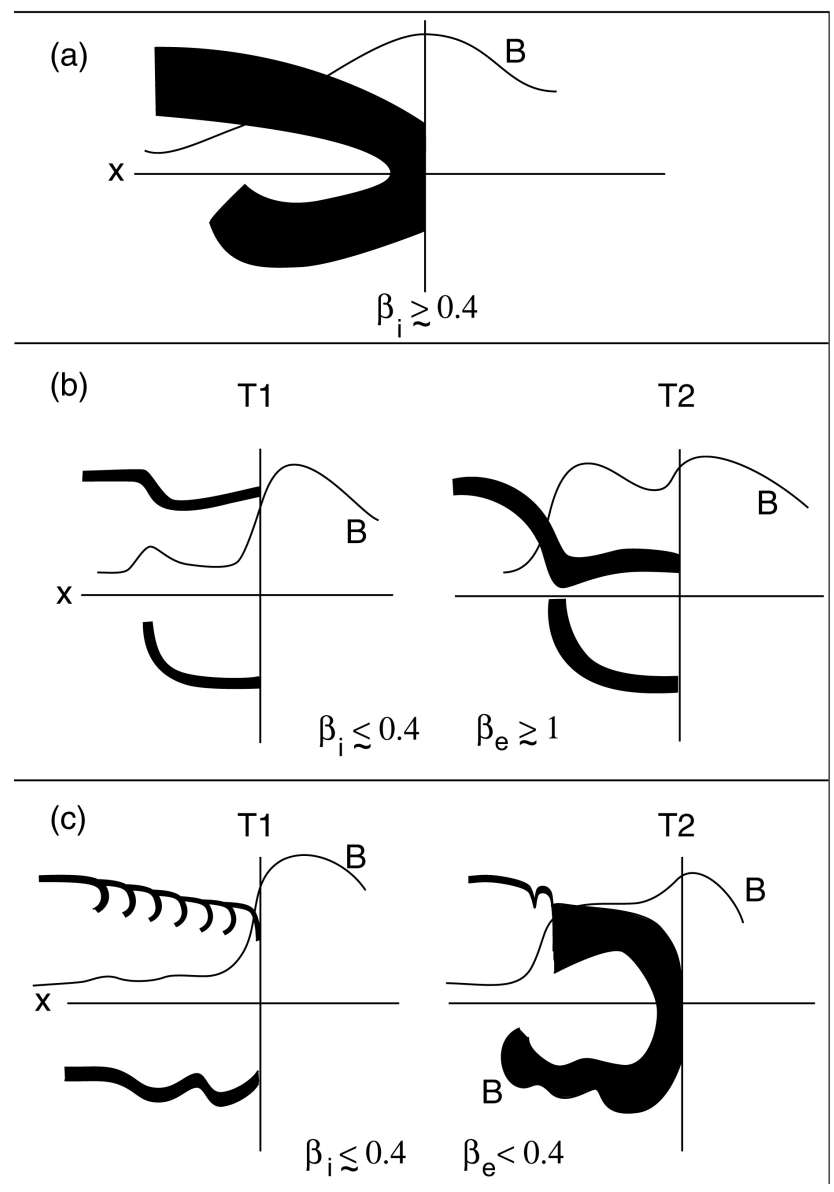

Fig. 12. Schematic of the magnetic field profile and of the ion phase space distribution at a medium Mach number nondispersive quasiperpendicular shock for various ion and electron beta conditions.

of reflected ions some distance upstream where the reflected ions are turned around and the velocity parallel to the shock normal goes to zero. The magnetic field increases at this upstream position, which, in turn, decelerates the incoming solar wind. This leads to a further density increase and eventually a new ramp arises. Since this process does not rely on a kinetic instability of the electrons it can be described by a massless electron fluid, as in the model by Hada et al. (2003). At the realistic mass ratio and low ion beta the modified twostream instability grows within the time the reflected ions need to travel from the ramp upstream before being turned around by the magnetic field. Once the waves have a large enough amplitude, they scatter the reflected ions and the incoming solar wind ions, which results in a hot distribution. The electrons are accelerated and trapped parallel to the magnetic field. This process proceeds from the ramp, where the wave amplitude is largest, towards the upstream edge of the foot. This demonstrates the imortance of the MTSI for the reformation process. A new shock ramp emerges at the upstream edge of the foot region when the solar ions and reflected ions are mixed in phase space. 
The process of electron heating can depend on the mass ratio: as shown above the heating occurs in different directions, depending on which mass ratio is used. This has to be taken into account when investigating electron heating processes with low mass ratio 2-D full particle simulations. Parallel electron heating occurs, of course, only when a modified two-stream instability is excited. In a different parameter regime, like higher ion beta or at less oblique, dispersive shocks, respectively, other heating mechanisms may be dominant (Savoini and Lembège, 1994).

Figure 12 is a suggested schematic of the structure of medium Mach number shocks. Shown is the magnetic field profile and the ion phase space distribution versus $x$. The upper panel (a) demonstrates the situation of a steady shock at high ion beta. The lower panel (c) shows the shock structure for a low ion and electron beta shock at two different times, $T_{1}$ and $T_{2}$. In the case of a low ion beta, but high electron beta shock the MTSI will either be stabilized or the growth rate smaller that an inverse ion gyrofrequency. Reformation would then proceed as in the Hada et al. (2003) model. This case is depicted in the middle panel (b).

We end the paper with the traditional caveat: these simulations are one-dimensional in space and all wave vectors $\boldsymbol{k}$ are forced in the shock normal direction. Instabilities with $\boldsymbol{k}$ vectors less oblique to the magnetic field may have larger growth rates and can modify the reformation process. In particular, the modified two-stream instability between solar wind electrons and reflected ions with $\boldsymbol{k}$ vector components parallel to the magnetic field can occur in a multi-dimensional spatial system (Gary et al., 1987) .

At present, computer resources do not allow twodimensional simulations with a mass ratio 1840 up to $\sim \Omega_{c i}^{-1}=3$. However, two-dimensional runs of a three component plasma, i.e. two ion beams and an electron background in a double periodic system could be run for smaller times. This setup can be taken as a proxy for the situation in the foot region of a quasi-perpendicular shock. The density of the reflected beam can be taken from the one-dimensional runs. Such a simulation can answer questions concerning the 2-D structure of the instability discussed here and concerning the importance of the electron/reflected ion instability relative to the electron/incoming ion instability. The second question concerns the use of an unrealistic small value of $\omega_{p e} / \Omega_{c e}$, at least as far the solar wind is concerned. The one-dimensional simulations have to be repeated by decreasing the mass ratio to a value where the two-stream instability is still dominant and increasing $\omega_{p e} / \Omega_{c e}$ to a value $\gg 1$. We have explored in the present paper one point in parameter space. However, in this case neither upstream accumulation of reflected ions nor a catastrophic development of a nonlinear upstream whistler train seems to be responsible for shock reformation, whereas the MTSI in the foot can explain the reformation process. In other parameter regimes different processes may be at work.

At the Earth's bow shock the inverse ion gyrofrequency is of the order of seconds. In order to observe the reformation process by multiple spacecraft the separation should not be larger than the distance the bow shock moves within a few seconds, i.e. rather short separation distances are required. Identification of the MTSI requires high resolution of the electric field normal component and of the ion density in foot region. The latter can possibly be obtained from spacecraft potential measurements.

Acknowledgements. The authors are grateful to D. Biskamp, B. Lembège, and R. A. Treumann for helpful discussions. The numerical code was provided by I. Shinohara. This work was supported in part by INTAS-01-0270.

Topical Editor T. Pulkkinen thanks two referees for their help in evaluating this paper.

\section{References}

Biskamp, D. and Welter, H.: Ion heating in high-Mach number, oblique collisionless shock waves, Nuclear Fusion, 12, 663-666, 1972.

Buneman, O.: Instability, turbulence, and conductivity in currentcarrying plasma, Phys. Rev. Lett., 1, 8-9, 1958.

Gary, S. P., Tokar, R. L., and Winske, D.: Ion/ion and electron/ion cross-field instabilities near the lower hybrid frequency, J. Geophys. Res., 92, 10 029-10 038, 1987.

Hada, T., Oonishi, M., Lembège, B., and Savoini, P.: Shock front nonstationarity of supercritical perpendicular shocks, J. Geophys. Res., 108 (A16), 1233, doi:10.1029/2002JA009339, 2003.

Kennel, C., Edmiston, J. P., and Hada, T.: In Collisionless shocks in the heliosphere: A tutorial review, edited by Stone, R. G. and Tsurutani, B. T., Geophys. Monographs 34, American Geophys. Union, Washington, D.C., 1-36, 1985.

Krasnoselskikh, V. V., Lembège, B., Savoini, P., and Lobzin, V. V.: Nonstationarity of strong collisionless quasiperpendicular shocks: Theory and full particle simulations, Phys. Plasmas, 9, 1192-1209, 2002.

Lembège, B. and Dawson, J. M.: Self-consistent study of a perpendicular collisionless and nonresistive shock, Phys. Fluids, 30, 1767-1788, 1987.

Lembège, B. and Savoini, P.: Non-stationarity of a two-dimensional quasi-perpendicular supercritical collisionless shock by selfreformation, Phys. Fluids, 4, 3533-3548, 1992.

Lembège, B., Savoini, P., Balikhin, M., Walker, S., and Krasnoselskikh, V.: Diamagnetization of transmitted electrons through a quasi-perpendicular collisionless shock, J. Geophys Res., 108 (A16), 1256, doi:10.1029/2002JA009288, 2003.

Liewer, P. C., Deceyk, V. K., Dawson, J. M., and Lembége, B.: Numerical studies of electron dynamics in oblique, qusiperpendicular collisionless shock waves, J. Geophys Res., 96, 9455-9465, 1991.

Matsukiyo, S. and Scholer, M.: Modified two-stream instability in the foot of high Mach number quasi-perpendicular shocks, J. Geophys. Res., 109 (A12), 1459, doi:10.1029/2003JA010080, 2003.

Savoini, P. and Lembège, B.: Electron dynamics in 2-dimensional and 1-dimensional supercritical magnetosonic shocks, J. Geophys Res., 99, 6609-6635, 1994.

Savoini, P. and Lembège, B.: Two-dimensional simulations of a curved shock: Self-consistent formation of the electron foreshock, J. Geophys Res., 106, 12 975-12 992, 2001.

Schmitz, H., Chapman, S. C., and Dendy, R. O.: The influence of electron temperature and magnetic field strength on cosmic-ray 
injection in high Mach number shocks, Astrophys. J., 570, 637646, 2002

Scholer, M., Shinohara, I., and Matsukiyo, S.: Quasi-perpendicular shocks: Length scale of the cross-shock potential, shock reformation, and implication for shock surfing, J. Geophys. Res., 108 (A1), 1014, doi:10.1029/2002JA009515, 2003.
Sckopke, N., Paschmann, G., Bame, S. J., and Russell, J. T.: Evolution of ion distributions across the nearly-perpendicular bow shock - Specularly and non-specularly reflected-gyrating ions, J. Geophys Res., 88, 6121-6136, 1983.

Shimada, N. and Hoshino, M.: Strong electron acceleration at high Mach number shock waves: Simulation study of electron dynamics, Astrophys. J., 543, L67-L71, 2000. 